\title{
Hospital care for children and young adults in the last year of life: a population-based study Chris Feudtner*1,2, David L DiGiuseppe ${ }^{3}$ and John M Neff ${ }^{4}$
}

\begin{abstract}
Address: ${ }^{1}$ Pediatric Advanced Care Team and the Pediatric Generalists Research Group, Division of General Pediatrics, Children's Hospital of Philadelphia, Philadelphia, Pennsylvania, USA, ${ }^{2}$ Center for Bioethics, Leonard Davis Institute of Health Economics, and Center for Clinical Epidemiology and Biostatistics, University of Pennsylvania, Philadelphia, Pennsylvania, USA, ${ }^{3}$ Child Health Institute, University of Washington, Seattle, Washington, USA and ${ }^{4}$ Center for Children with Special Needs, Children's Hospital and Regional Medical Center; Department of Pediatrics, University of Washington; Seattle, Washington, USA
\end{abstract}

Email: Chris Feudtner* - feudtner@email.chop.edu; David L DiGiuseppe - davdig@u.washington.edu; John M Neff - john.neff@seattlechildrens.org

* Corresponding author

Published: 23 December 2003

BMC Medicine 2003, 1:3

This article is available from: http://www.biomedcentral.com/174I-70I5/I/3
Received: 08 October 2003

Accepted: 23 December 2003

(C) 2003 Feudtner et al; licensee BioMed Central Ltd. This is an Open Access article: verbatim copying and redistribution of this article are permitted in all media for any purpose, provided this notice is preserved along with the article's original URL.

\begin{abstract}
Background: To help design population-based pediatric palliative care services, we sought to describe the hospital care received in the last year of life by children and young adults who died. We also determined the proportion with complex chronic conditions (CCCs) and tested whether the use of hospital services increased as the date of death drew nearer.

Methods: For all deaths occurring under 25 years of age from 1990 to 1996 in Washington State, USA, we linked death certificate information to hospital utilization records and analyzed the timing and duration of hospitalizations and the nature of hospital procedures during the year prior to death.

Results: Of the 8893 deaths, $25 \%$ had CCCs. Among infants with CCCs, $84 \%$ were hospitalized at the time of death and $50 \%$ had been mechanically ventilated during their terminal admission. Among the 458 CCC neonates dying under a week of age, $92 \%$ of all days of life were spent in the hospital; among the 172 CCC neonates dying during the second to fourth weeks of life, $85 \%$ of all days of life were spent hospitalized; among the 286 CCC infants dying during the second to twelfth month of life, $41 \%$ of all days of life were spent hospitalized. Among children and young adults with CCCs, $55 \%$ were hospitalized at the time of death, and $19 \%$ had been mechanically ventilated during their terminal admission. For these older patients, the median number of days spent in the hospital during the year preceding death was 18 , yet less than a third of this group was hospitalized at any point in time until the last week of their lives. The rate of hospital use increased as death drew near. For subjects who had received hospital care, $44 \%$ had governmental insurance as the source of primary payment.

Conclusions: Infants who died spent a substantial proportion of their lives in hospitals, whereas children and adolescents who died from CCCs predominantly lived outside of the hospital during the last year of life. To serve these patients, pediatric palliative and end-of-life care will have to be provided in an integrated, coordinated manner both in hospitals and home communities.
\end{abstract}




\section{Background}

What hospital care do children and young adults who die receive in the last year of their lives? Answering this question would help us to better envision and organize the emerging system of pediatric palliative, end-of-life, and bereavement (P-EOL-B) care. The American Academy of Pediatrics recently stated that "palliative care and respite programs need to be developed and widely available" in order to meet the needs of "children living with lifethreatening or terminal conditions" [1]. The need to develop programs and reorganize the health care system were also key themes in the Institute of Medicine's report, When Children Die: Improving Palliative and End-of-Life Care for Children and Their Families, which asserted that "better care is possible now, but current methods of organizing and financing palliative, end-of-life, and bereavement care complicate the provision and coordination of services to help children and families ..." [2]. While both reports recommended improving the techniques of P-EOL-B care delivered to individual patients and families, they also recommended innovation at the level of health care systems, aiming to improve the delivery of competent, compassionate, comprehensive, coordinated and continuous care, and to assure that reimbursement systems effectively incentivize excellent care $[1,2]$.

If we knew the nature and timing of the hospital care services that children receive prior to their deaths, we would be better able to identify health care system-level opportunities and challenges to improving the quality of pediatric P-EOL-B care. Accordingly, the IOM report, in discussing directions for future research, called for more epidemiologic studies documenting the circumstances surrounding deaths during childhood. For instance, determining the proportion of deaths that were preceded by some period of hospitalization and whether these hospitalizations occurred throughout the year or were concentrated in only the very last period of life, would begin to address the potential impact of hospital-based P-EOL-B services on the entire population of children who die and their families.

Our current population-based knowledge of pediatric end-of-life experience, however, is severely limited, with few studies conducted beyond the confines of institutions [3-5]. One study found that between 1980 and 1998 in Washington State, USA, deaths among children and young adults with complex chronic conditions (CCCs) were almost entirely occurring in hospitals if the child was under a year of age, and if older than a year of age were increasingly occurring at home, with a greater probability of a home death if the child resided in a more affluent local area [6]. Descriptive case series from single hospitals [7-10] or hospices [11] have also been published, but these have captured the experience of a small and perhaps non-representative proportion of all dying children. Even for adults, few population-based studies have explored the hospital care received prior to death, and these have been limited to people who died beyond their $65^{\text {th }}$ birthday or died after having Alzheimer's disease or some other form of dementia [12-16]. Any plan for a system of pediatric P-EOL-B care is therefore potentially compromised by inadequate information regarding the characteristics of dying children and the care they are likely to receive.

We therefore sought to create a population-level portrait of the nature and timing of hospital care received by children and young adults who subsequently died. We focused particularly on those individuals who died with an underlying CCC, as this subpopulation could benefit substantially from improvements in P-EOL-B care. Since some of these young adults with CCCs continue to receive care from pediatricians at pediatric facilities until the middle of their third decade of life, we extended the age range of our analysis, including CCC-related deaths from birth up to the twenty-fifth birthday. Finally, since - based on anecdotal evidence - discussions regarding palliative and end-of-life care are often delayed until a child with a CCC becomes ill enough to warrant hospitalization, we sought to determine the degree to which hospital utilization increased as the date of death drew nearer.

\section{Methods}

The institutional review boards for both the Washington State Department of Health and the University of Washington approved the design and conduct of this study.

\section{Data sources and linkage}

The Washington State Department of Health assembled the longitudinal Death and Illness History Database (DIHD), which comprises Washington State hospital inpatient discharge records linked to death certificate records for all people who died in Washington State from 1987 to 1996. The Office of Hospital and Patient Data Systems maintains the hospital discharge records as Comprehensive Hospital Abstract Reporting System (CHARS) data, and the Center for Health Statistics maintains the death certificate files. The CHARS data does not contain records of emergency department utilization without admission to the hospital. Deaths of patients in emergency departments, while noted on the death certificate, would not have been recorded in the CHARS data. Concerns for the quality of data during the initial years of 1987 and 1988 led us to restrict the analysis to deaths that occurred from 1990 to 1996, with linkage to CHARS records from 1989 to 1996.

Each death certificate was linked to the most recent hospital inpatient discharge record for a given person. The data linkage occurred in seven steps, using various 
combinations of birth date, gender, the first two letters of the last and first name, residential zip code, hospital where death occurred, code indicating that patient died, and date of death equal to or greater than date of discharge. Subsequent to the linkage, the CHARS unique patient identifiers were used to append to the DIHD file all CHARS hospital discharge records for people with successful death certificate linkages. Some infant death certificates that were linked to the most recent CHARS record appeared not to have been linked to the corresponding hospital birth records (that is, the earliest hospitalization record). We suspect that this was due to changes in the first name or surname of the child during the first days of life, which would have precipitated a change in such a child's unique CHARS patient identifier. Therefore, to be included in the final analytic data set, infants who died under 1 year of age had to have a discharge record that incorporated the newborn period. If this discharge record contained DRG code 391 for "normal newborn" and a length of stay of less than four days, then this hospitalization was not counted as a hospitalization per se in subsequent analyses (since our intention was to study hospitalizations related to illness and not simply the event of being born). If the birth hospitalization record indicated any abnormalities, it was classified as the first hospitalization.

Given the lack of an external criterion standard by which to assess the thoroughness of the linkage of CHARS records to death certificates (since not every death certificate need have a corresponding set of CHARS records, nor vice versa), we assessed the linkage process by first determining the proportion of cases in the DIHD where a CHARS record documented 'death' as the discharge disposition and was linked to a corresponding death certificate record $(99.5 \%)$ and then assessing the proportion of cases where a death certificate recorded the site of death as 'hospital' and was linked to a corresponding CHARS record $(69 \%)$. Among the $31 \%$ of cases where the site of death was listed as the 'hospital' on the death certificate but there was no corresponding CHARS record, $32 \%$ died due to trauma (compared to $21 \%$ of the cases with a death certificate record-to-CHARS match), leading us to postulate that some fraction of these unmatched cases represent cases that expired in the emergency department and were miscoded as 'hospital' death on the death certificate (when they should have been coded as 'emergency department' for the site of death). We presume, however, that some portion of these unmatched cases truly died in the hospital but were unmatched to CHARS data. Consequently, since our determination of hospital services received was based on the presence of CHARS records and not on the place of death field on the death certificate, our results are probably conservative, in that they are likely biased towards underreporting the true extent of hospital services received by these cases.

\section{Classification of complex chronic conditions}

We have defined CCCs as any medical condition that can be reasonably expected to last at least 12 months (unless death intervenes) and to involve either several different organ systems or one organ system severely enough to require specialty pediatric care and probably some period of hospitalization in a tertiary care center. Although prematurity, low-birth weight and complications of pregnancy constitute a large proportion of the cited underlying cause of death during the neonatal period, these conditions are not considered CCCs because they often remit and are not uniformly chronic conditions. Based on this definition and several published lists of ICD-9 codes from earlier studies that examined health service utilization for children with congenital or complicated medical conditions, we mapped specific ICD-9 codes to nine CCC diagnostic categories: neuromuscular, cardiovascular, respiratory, renal, gastrointestinal, hematological or immunological, metabolic, malignancy, and genetic or other congenital defect conditions $[17,18]$

\section{Statistical analyses}

We began by calculating proportions and central tendencies for univariate characteristics, including gender, age, race and ethnicity, underlying cause of death, hospital procedures, length of hospital stay, and site of death. We then calculated three additional parameters that have implications for the identification of patients and delivery of palliative care services through hospital-based systems. First, we counted the number of days in the time interval either from the date of birth to the first observed non-normal-newborn hospitalization (for infant deaths), or (for deaths occurring beyond the first birthday) from 365 days prior to death to the date of the first observed hospitalization. Second, we counted the number of days each case was hospitalized in the year prior to death. Third, we also calculated the proportion of these patients who were hospitalized for each of the 365 days prior to death.

To determine whether the rate of hospitalization increased during the last year of life as the date of death drew closer among cases that were older than a year of age at the time of death, we employed negative binomial regression modeling. Each case contributed a control period consisting of the first six months of the last year of life (that is, from 12 months prior to death to 7 months prior to death), with the rate of hospitalizations during this interval constituting the baseline rate, which was then compared to the rate of hospitalization for each of the ensuing six one-month intervals, expressed as relative rate ratios with $95 \%$ confidence intervals. The model adjusted for each case's age at the date of death, and accounted for 
Table I: Demographics of subjects, 1990-1996

\begin{tabular}{|c|c|c|}
\hline & Number & Percentage \\
\hline \multicolumn{3}{|l|}{ Sex } \\
\hline Male & 5,906 & 66.4 \\
\hline Female & 2,987 & 33.6 \\
\hline TOTAL & 8,893 & 100.0 \\
\hline \multicolumn{3}{|l|}{ Age at death } \\
\hline I month or less & 1,493 & 16.8 \\
\hline 2-II months & 1,038 & 11.7 \\
\hline $\mathrm{I}-4$ years & 841 & 9.5 \\
\hline $5-9$ years & 530 & 6.0 \\
\hline $10-14$ years & 634 & 7.1 \\
\hline $15-24$ years & 4,357 & 49.0 \\
\hline TOTAL & 8,893 & 100.0 \\
\hline \multicolumn{3}{|l|}{ Race or Ethnicity } \\
\hline White, non-Hispanic & 6,529 & 73.4 \\
\hline Black, non-Hispanic & 646 & 7.3 \\
\hline Asian or Pacific Islander & 401 & 4.5 \\
\hline Native American & 338 & 3.8 \\
\hline Hispanic & 959 & 10.8 \\
\hline Other or Unknown & 20 & 0.2 \\
\hline TOTAL & 8,893 & 100.0 \\
\hline \multicolumn{3}{|l|}{ Underlying Cause } \\
\hline Prematurity or IRDS & 242 & 2.8 \\
\hline SIDS & 265 & 3.0 \\
\hline Trauma & 3,873 & 43.6 \\
\hline \multicolumn{3}{|l|}{$\mathrm{CCC}$} \\
\hline Cardiovascular & 557 & 6.3 \\
\hline Neuromuscular & 376 & 4.2 \\
\hline Malignancy & 613 & 6.9 \\
\hline Respiratory & 201 & 2.3 \\
\hline Renal & 63 & 0.7 \\
\hline $\mathrm{Gl}$ & 26 & 0.3 \\
\hline Hematologic/lmmunologic & 44 & 0.5 \\
\hline Genetic/Congenital & 251 & 2.8 \\
\hline Metabolic & 52 & 0.6 \\
\hline Multiple CCC Categories & 78 & 0.9 \\
\hline Other Cause & 2,252 & 25.3 \\
\hline TOTAL & 8,893 & 100.0 \\
\hline
\end{tabular}

the clustering of observations on individual cases. The model was fitted using the set of all cases classified as having a CCC, as well as for each of the CCC subgroups.

For all statistical analyses we used Stata 7.0 [19]. The Stata software commands that replicate the analysis are available in PDF format; see additional file 1: Analysis_Commands.pdf.

\section{Results}

Our match of death certificates with hospital discharge records identified 8893 persons less than 25 years of age who died in Washington State between 1 January 1990 and 31 December 1996 (Table 1). Two-thirds of the children and young adults were male, and over a fourth were under a year of age at the time of death. While trauma was the leading cause of death, a quarter of all individuals had a CCC identified as the underlying cause of death. Among those fatalities that were associated with a prior hospitalization, $44 \%$ were identified as having a governmental insurance source of primary payment (Table 2).

\section{What hospital services or procedures are used prior to death?}

Infants who died differed markedly from older patients who died in terms of the use of hospital services or procedures (Table 3). For infants, $79 \%$ were hospitalized (for some reason other than a normal newborn delivery) at some point during their lives, whereas only $35 \%$ of the older children and young adults were hospitalized during 
Table 2: Primary payer identified among subjects hospitalized during the year prior to death.

\begin{tabular}{|c|c|c|c|}
\hline \multicolumn{4}{|c|}{$\underline{\text { CCC Diagnosis }}$} \\
\hline & $\underline{\text { Yes }}$ & No & Total \\
\hline Primary Payer & $\%$ & $\%$ & $\%$ \\
\hline Medicaid & 40.2 & 39.9 & 40.0 \\
\hline Medicare & 1.9 & 0.5 & 1.0 \\
\hline Other Governmental & 2.9 & 3.3 & 3.2 \\
\hline HMO & 14.5 & 8.6 & 10.7 \\
\hline Private Insurance & 37.1 & 35.8 & 36.3 \\
\hline Worker's Compensation & 0.1 & 0.4 & 0.3 \\
\hline Self Pay & 3.3 & 11.6 & 8.6 \\
\hline Charity Care & 0.1 & 0.1 & 0.1 \\
\hline TOTAL & 100.0 & 100.0 & 100.0 \\
\hline
\end{tabular}

Table 3: Utilization of hospital services or procedures within the last year of life, Washington State, USA 1990 - 1996.

\begin{tabular}{|c|c|c|c|c|c|c|c|c|}
\hline & \multicolumn{4}{|c|}{ ALL DEATHS } & \multicolumn{4}{|c|}{ DEATHS WITH CCCs } \\
\hline & \multicolumn{2}{|c|}{ Age $<$ I } & \multicolumn{2}{|c|}{ Age $>=1$} & \multicolumn{2}{|c|}{ Age $<$ I } & \multicolumn{2}{|c|}{ Age $>=1$} \\
\hline & $\underline{\mathrm{n}}$ & $\underline{\%}$ & $\underline{\mathrm{n}}$ & $\underline{\%}$ & $\underline{\mathrm{n}}$ & $\underline{\%}$ & $\underline{\mathrm{n}}$ & $\underline{\%}$ \\
\hline Total number of deaths & 2,531 & 100 & 6,362 & 100 & 916 & 100 & 1,345 & 100 \\
\hline Any Inpatient Hospital Care & 1,991 & 79 & 2,217 & 35 & 916 & 100 & 1,123 & 84 \\
\hline Terminal Hospitalization* & 1,540 & 61 & $\mathrm{I}, 576$ & 25 & 769 & 84 & 737 & 55 \\
\hline Any Mechanical Ventilation** & 990 & 39 & 759 & 12 & 512 & 56 & 329 & 25 \\
\hline $\begin{array}{l}\text { During Terminal } \\
\text { Hospitalization* }\end{array}$ & 911 & 36 & 670 & 11 & 462 & 50 & 259 & 19 \\
\hline Any Other Procedure & $\mathrm{I}, 147$ & 45 & $\mathrm{I}, 835$ & 29 & 627 & 69 & 945 & 70 \\
\hline $\begin{array}{l}\text { During Terminal } \\
\text { Hospitalization* }\end{array}$ & 879 & 35 & 1,248 & 20 & 505 & 55 & 525 & 39 \\
\hline
\end{tabular}

* Terminal hospitalizations were defined as any period of hospitalization that terminated on the date of the patient's death. ** Includes any assisted ventilation, any mechanical ventilation, and any insertion of an endotracheal tube.

the last year of life. Two-thirds of the infants were hospitalized at the time of death, while only a quarter of the older cases were. Just over a third of infants were mechanically ventilated at some point during their lives, while only $12 \%$ of older cases received mechanical ventilation during their last year of life. Aside from mechanical ventilation, $45 \%$ of infants and $29 \%$ of older cases received some other procedure.

Among infants, children, and young adults that had CCCs identified as the underlying cause of death, compared to all deaths from all causes, larger proportions of both infants $(100 \%)$ and older cases ( $84 \%)$ were hospitalized at some point during their last year of life, with $84 \%$ of infants and $55 \%$ of older cases dying at the conclusion of what proved to be a terminal hospitalization (Table 3). Mechanical ventilation was provided to $56 \%$ of these infants and $25 \%$ of the older cases, while roughly $70 \%$ of both infants and older cases underwent some other surgical or medical procedure.

\section{When are patients who die hospitalized?}

Hospital-based P-EOL-B programs, endeavoring to identify patients who would benefit from enhanced supportive care services, might use a case-finding system that focused on hospitalized patients. Ideally, this system - or any such system - would identify patients sooner rather than later. We therefore examined the timing of the first hospitalization during the last year of life (Table 4). For young neonates, a hospital-based case-finding system conceivably could identify the majority of these patients in a timely manner, since $89 \%$ of neonates who died under a week of age were hospitalized by the first day of life, and $74 \%$ of neonates who died between one and four 
Table 4: Timing of first hospitalization during the last year of life.

\begin{tabular}{|c|c|c|c|c|c|c|c|c|c|c|c|c|c|c|c|c|c|}
\hline \multirow{3}{*}{$\begin{array}{r}\text { TIME } \\
\text { INTERVAL * } \\
\text { Day: } \\
\text { Week: }\end{array}$} & & ROPC & TION & F INL & IDUA & WH & HAD & HEIR & ST H & SPIT/ & IZAT & N DL & NG 7 & E IN & VAL * & & TOTAL \\
\hline & I & 2 & $3-7$ & & & & & & & & & & & & & & \\
\hline & & & & 2 & $3-4$ & & & & & & & & & & & & \\
\hline Month: & I & & & & & 2 & 3 & 4 & 5 & 6 & 7 & 8 & 9 & 10 & II & 12 & \\
\hline $\begin{array}{l}\text { AGE AT } \\
\text { DEATH }\end{array}$ & $\%$ & $\%$ & $\%$ & $\%$ & $\%$ & $\%$ & $\%$ & $\%$ & $\%$ & $\%$ & $\%$ & $\%$ & $\%$ & $\%$ & $\%$ & $\%$ & $\%$ \\
\hline \multicolumn{18}{|l|}{ All Individuals } \\
\hline$<$ I week & 88.5 & 5.3 & 0.4 & & & & & & & & & & & & & & 94.1 \\
\hline I-4 weeks & 73.7 & 4.4 & 1.8 & 3.5 & 2.1 & & & & & & & & & & & & 85.4 \\
\hline $1-12$ months & 47.4 & 2.0 & 1.5 & 0.7 & 1.5 & 2.5 & 1.6 & 1.0 & 0.3 & 0.4 & 0.4 & 0.1 & 0.1 & 0.0 & 0.0 & 0.2 & 59.7 \\
\hline > I year & 2.1 & & & & & 1.6 & 1.4 & 1.4 & 1.3 & 1.6 & 1.4 & 1.0 & 1.6 & 1.8 & 1.9 & 17.8 & 34.9 \\
\hline \multicolumn{18}{|l|}{$\begin{array}{l}\mathrm{CCC} \\
\text { Individuals }\end{array}$} \\
\hline$<$ I week & 92.6 & 6.8 & 0.7 & & & & & & & & & & & & & & 100 \\
\hline I-4 weeks & 87.2 & 6.4 & 1.7 & 4.1 & 0.6 & & & & & & & & & & & & 100 \\
\hline $1-12$ months & 80.4 & 5.9 & 2.5 & 1.4 & 1.4 & 2.8 & 2.8 & 1.1 & 0.4 & 0.4 & 0.7 & 0.0 & 0.4 & 0.0 & 0.0 & 0.0 & 100 \\
\hline$>$ I year & 8.1 & & & & & 5.4 & 5.1 & 4.6 & 4.8 & 5.5 & 5.1 & 3.4 & 4.8 & 6.3 & 6.5 & 23.9 & 83.5 \\
\hline
\end{tabular}

* For individuals who died during infancy, the time interval refers to when the first hospitalization occurred, measured in days, weeks, or months of life. For individuals who died past infancy, the time interval refers to when the first hospitalization occurred during the last year of life, measured in months counted from I (12 months prior to death) to 12 (the month in which death occurred).

Table 5: Days spent in the hospital during the last year of life

\begin{tabular}{rcccccccccccc}
\hline \multicolumn{10}{c}{ PROPORTION OF CASES HOSPITALIZED FOR SPECIFIED NUMBER OF DAYS DURING LAST YEAR OF } \\
LIFE \\
\hline
\end{tabular}

weeks of life were also hospitalized by the first day of life. Among the neonates with underlying CCCs, these proportions were even higher. For infants that died between one and 12 months of age, the majority were hospitalized within the first week of life (51\%), whereas $89 \%$ of those with CCCs were hospitalized within the first week of life.

For deaths occurring beyond infancy, a hospitalizationbased case-finding system would detect a much smaller proportion of all fatal cases (35\%), with $18 \%$ detected only in the last month of life. Among children and young adults with underlying CCCs, $84 \%$ would conceivably be identified, with $24 \%$ detected only during the last month of life.

\section{Does the rate of hospital admissions increase towards the end of life?}

Another potential method to locate children who might benefit from palliative care would be to examine the rate of hospital admissions for an individual patient, on the premise that many patients nearing the end of life utilize more services. Chronically ill patients admitted to the hospital with increasing frequency could potentially be targeted for more intensive evaluation of the appropriateness of P-EOL-B services. To assess the feasibility of using 


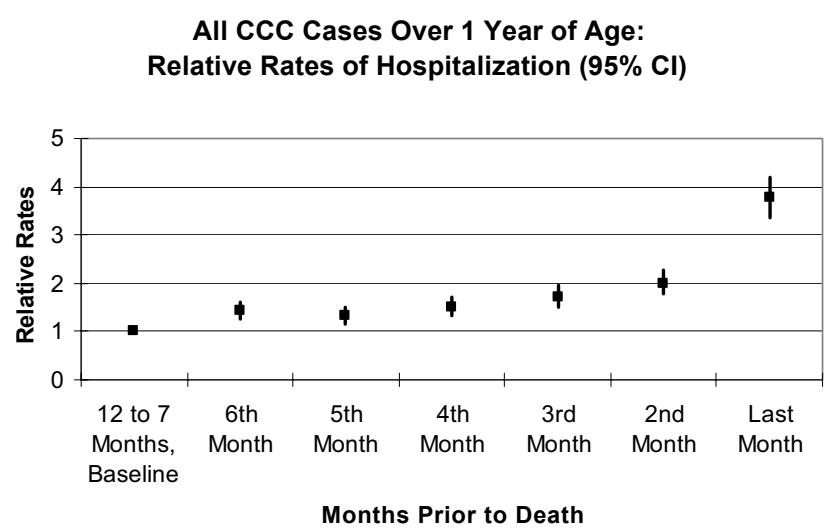

Figure I

Rate of admissions over the last year of life.

frequency of admission as a patient-specific predictor, we examined the rates of admission over time across all patients with CCCs and within CCC subgroups.

The experience of CCC patients in this cohort who were older than a year of age at the time of death suggests that such a scheme may not give sufficiently advanced notice of impending death (Fig. 1). Using the period from 12 to seven months prior to death as a baseline, and adjusting for the age of the child or young adult at the date of death, the rate of hospitalization among all CCC cases increased gradually to twice the baseline rate by the second-to-last month of life, and then to four times the baseline rate during the last month of life. For individuals where death was due to cancer, the rate in the last month rose to only 2.4 times greater than baseline (with the unadjusted absolute rates of admission rising from 28 admissions per 100 person-months during the baseline period to 70.4 admissions per 100 person-months during the last month of life). For individuals with neurological CCCs, the final month's rate of hospitalization was seven-fold greater than baseline (unadjusted absolute rates from 8.7 to 58.4 admissions per 100 person-months), while for cardiac CCC patients, the rate rose nine-fold (from 5.6 to 52.2 per 100 person-months).

How much time do patients who die spend in the hospital? Some infants, children and adolescents who died spent substantial amounts of their last year of life hospitalized, measured either as a proportion of their lifespans (which is a particularly pertinent patient- and family-centered measure for infants who die early in life) or as a count of the number of days spent in the hospital (which reveals the amount of hospital use, as is shown in Table 5). Among CCC cases, measuring the duration of hospitaliza-

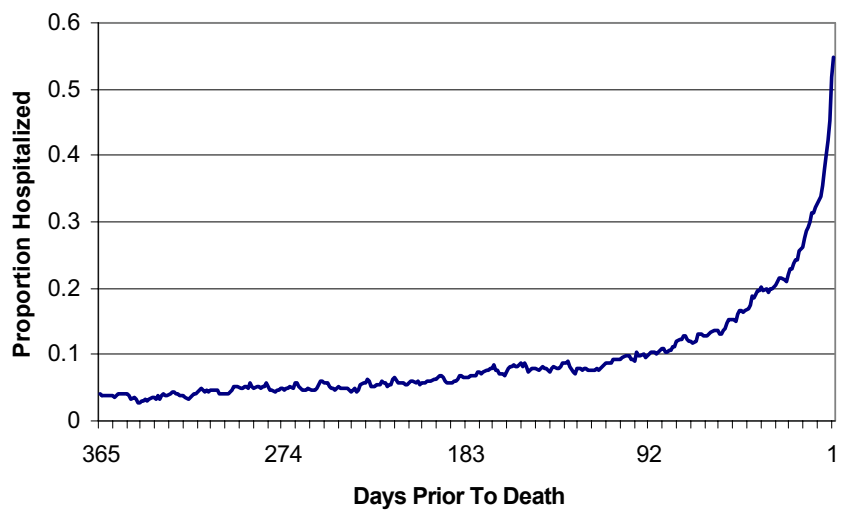

Figure 2

Proportion of children who subsequently died from a CCC hospitalized during the preceding one-year interval.

tion as a proportion of their entire lifespan, $92 \%$ of neonates who died under a week of age spent their entire lives hospitalized, $85 \%$ of neonates who died between one and four weeks of age spent their entire lives hospitalized, and $41 \%$ of infants who died between one and 12 months spent their entire lives hospitalized. Again focusing on the CCC cases, but now looking at the absolute duration of hospitalization measured in days, $59 \%$ of those that died between one and 12 months of age spent over a month in the hospital, while $39 \%$ of the children who died after a year of age spent over a month in the hospital. Among these older CCC cases, the median number of days spent in the hospital during the preceding year was 18 and the $75^{\text {th }}$ percentile was 52 days.

Looking at the entire potential target population of chronically ill children and adolescents who will subsequently die, however, the hospital is never where they are most likely to be found. For the CCC cases that died after a year of age, less than $10 \%$ were hospitalized on any given day until they had less than three months to live, and only during the last week of life were more than a third hospitalized (Fig. 2). When the hospitalized proportion of a population is viewed as presented by Fig. 2, the area under the curve reflects the proportion of time that this population conceivably could be under the direct care of an inpatient palliative care service, with the area over the curve corresponding to the proportion of time when outpatient, community-based, or in-home services might be necessary.

\section{Discussion}

Examining the inpatient hospital care experience of all children who died in Washington State, USA from 1990 to 1996, we found that infants and cases with CCCs 
(compared to children older than a year of age and those cases without CCCs, respectively) were significantly more likely to be hospitalized and to experience mechanical ventilation or some other procedure, in general and specifically during the terminal admission. While most infants who died were hospitalized early in their lives, the majority of older children who died were either never hospitalized or only during the last six months of their lives (and often not until the last month of life). Among older children and young adults who died with a CCC, a substantial proportion spent weeks to months in the hospital during the last year of their lives, with the rate of hospital admission rising as the date of death drew closer. Nevertheless, on any given day during that last year of life, the majority of these older CCC cases were not in the hospital.

This population-based study of end-of-life care for children provides a valuable perspective due to the breadth of its surveillance, extending beyond the confines of a single institution or a restricted range of disease conditions. Although this is the first population-level study to focus on the hospital care experience of children who died, our analysis was nevertheless limited in several ways. Only services arising from a hospital admission were observed, leaving the question of what ambulatory, home-based, emergency medical, or emergency department services these children received unanswered. We also do not know, from the vantage of the patient, family or physicians, which of the services received were desired, nor which desired services were never received. Although unlikely to have substantially influenced our findings, the study design did not permit us to account for migration of cases into the state of Washington during the period of observation, so that we failed to observe hospital utilization in another state, or migration out from the state, so that we failed to observe an unknown number of deaths. The observation period is now nearly a decade old, raising the possibility that usage patterns have changed since that time. Finally, this analysis was limited to fatal cases only, precluding inferences about how fatal cases differed from non-fatal cases.

Keeping these limitations in mind, we believe that this study has several important implications regarding the "who, where, when, and how" of P-EOL-B care. First, although these services are often thought of in the context of school age children or teenagers with advanced cancer, the preponderance of childhood death occurs during infancy and the majority of deaths are never due to cancer. For infant patients who die with a CCC, a hospital-based service system may be well situated to deliver timely and continuous P-EOL-B services, given the likelihood of early and extensive hospitalization for these children. For older children with CCCs and their families, however, various outpatient, community-based, or at-home services - such

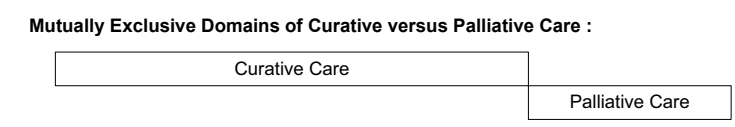

Alternative Domains of Curative versus Palliative Care:

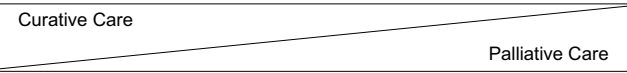

Complementary, Concurrent Components of Care: Cure-Seeking Care

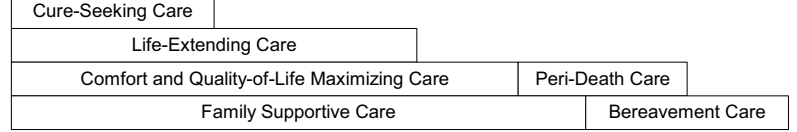

DIAGNOSIS Family Supportive Care DEATH TIME

Figure 3

Alternative models of how to provide palliative, end-of-life, and bereavement care.

as pain management, home nursing, hospice, or bereavement counseling - would typically be necessary to care for them when they are not hospitalized. Indeed, with $44 \%$ of all fatalities attributed to trauma cases that were associated with little or no hospital utilization, bereavement services are probably best conceived of as community-based with well-established ties to the emergency department, inpatient realm, and even the medical examiner's office. One element of a well-designed pediatric P-EOL-B care system would therefore be explicit relationships between health care facilities that care for sick and injured children, their available inpatient P-EOL-B services, and community-based services.

Second, the drastically increased tempo of hospitalization during the last month of life for children who died with certain underlying conditions, such as cardiac or neurological CCCs, suggests that the timely delivery of effective hospital-based P-EOL-B services will be greatly impaired if the means to prevent or relieve suffering are withheld until the other forms of medical care have been exhausted. As recommended by both the American Academy of Pediatrics statement and the Institute of Medicine report, a blended or complementary model of care - with the simultaneous delivery of different modes of care, from cure-seeking or life-extending to comfort-seeking or family-supportive - is how pediatric EOL care should ideally be delivered (Fig. 3). Determining ways to incentivize health care providers and institutions to deliver such complementary care (through improved financing arrangements, payment systems, and organizational culture), and then to measure the degree to which such complementary care is actually delivered and the subsequent outcomes for 
children and families, will be important steps towards improving the quality of the pediatric P-EOL-B system.

Finally, regarding the ethics of pediatric end-of-life care, much of the literature has focused on the questions of when and how to withdraw mechanical ventilation and other forms of life support. Certainly these decisions warrant scrupulous attention. Yet this study suggests that the withdrawal of mechanical ventilation may have happened - at most - in fewer than half of all the cases. This proportion conforms with that found in a study of all deaths occurring in 60 children's hospitals, in which approximately $50 \%$ of patients overall were mechanically ventilated during their terminal hospital admission [20]. While the manner in which intensive care is withdrawn should still receive thorough and thoughtful scrutiny, we propose that the ethical comportment of the pediatric PEOL-B care system would be enhanced by expanding the focus from individual clinical decisions to the broader issue of patients' and families' access to timely and effective P-EOL-B services, both within the walls of hospitals and within the context of their home communities.

\section{Conclusions}

Infants who died spent a substantial proportion of their lives in hospitals, whereas children and adolescents who died from CCCs predominantly lived outside of the hospital during the last year of life. To serve these patients, pediatric palliative and end-of-life care will have to be provided in an integrated, coordinated manner both in hospitals and home communities.

\section{Competing interests}

None declared.

\section{Authors' contributions}

$\mathrm{CF}$ conceived of and designed the study, obtained and assisted in analyzing the data, and drafted the manuscript. DD assisted in the design of the study, analyzed the data, and critically reviewed the manuscript. JN assisted in the design of the study and analysis of the data, and critically reviewed the manuscript. All authors read and approved the final manuscript.

\section{Additional material}

\section{Additional File 1}

Readers can retrieve a copy of the Stata software commands, available in PDF format in the file named "Analysis_Commands.pdf", that replicate our analysis.

Click here for file

[http://www.biomedcentral.com/content/supplementary/1741-

7015-1-3-S1.pdf]

\section{Acknowledgements}

This project was supported by grant number $\mathrm{K} 08 \mathrm{HS} 00002$ from the Agency for Healthcare Research and Quality. The authors thank Bill O'Brien and Thomas D. Koepsell, MD MPH for their assistance and suggestions.

\section{References}

I. American Academy of Pediatrics: Committee on Bioethics and Committee on Hospital Care. Palliative care for children. Pediatrics 2000, 106:35 I-357.

2. Field MJ, Behrman RE, editors: When Children Die: Improving Palliative and End-of-Life Care for Children and Their Families Washington, DC: National Academy Press; 2002

3. Wolfe J, Grier HE, Klar N, Levin SB, Ellenbogen JM, Salem-Schatz S, Emanuel EJ, Weeks JC: Symptoms and suffering at the end of life in children with cancer. $N$ Engl J Med 2000, 342:326-333.

4. Meyer EC, Burns JP, Griffith JL, Truog RD: Parental perspectives on end-of-life care in the pediatric intensive care unit. Crit Care Med 2002, 30:226-23I.

5. Drake R, Frost J, Collins JJ: The symptoms of dying children. J Pain Symptom Manage 2003, 26:594-603.

6. Feudtner C, Silveira MJ, Christakis DA: Where do children with complex chronic conditions die? Patterns in Washington State, 1980-1998. Pediatrics 2002, 109:656-660.

7. Ashby MA, Kosky RJ, Laver HT, Sims EB: An enquiry into death and dying at the Adelaide Children's Hospital: a useful model? Med J Aust 199I, 154:165-I70.

8. van der Wal ME, Renfurm LN, van Vught AJ, Gemke RJ: Circumstances of dying in hospitalized children. Eur J Pediatr 1999, I 58:560-565.

9. McCallum DE, Byrne P, Bruera E: How children die in hospital. J Pain Symptom Manage 2000, 20:417-423.

10. Pierucci RL, Kirby RS, Leuthner SR: End-of-life care for neonates and infants: the experience and effects of a palliative care consultation service. Pediatrics 200I, 108:653-660.

II. Goldman A, Beardsmore S, Hunt J: Palliative care for children with cancer - home, hospital, or hospice? Arch Dis Child 1990, 65:64I-643.

12. Roos NP, Montgomery P, Roos LL: Health care utilization in the years prior to death. Milbank $Q$ 1987, 65:23I-254.

13. Collins C, Ogle K: Patterns of predeath service use by dementia patients with a family caregiver. J Am Geriatr Soc 1994, 42:719-722.

14. Brock DB, Foley DJ, Salive ME: Hospital and nursing home use in the last three months of life. J Aging Health 1996, 8:307-319.

15. Liao Y, McGee DL, Cao G, Cooper RS: Quality of the last year of life of older adults: 1986 vs 1993 . JAMA 2000, 283:5।2-5I8.

16. Wilson DM, Truman CD: Does the availability of hospital beds affect utilization patterns? The case of end-of-life care. Health Serv Manage Res 200I, I4:229-239.

17. Feudtner C, Christakis DA, Connell FA: Pediatric deaths attributable to complex chronic conditions: a population-based study of Washington State, 1980-1997. Pediatrics 2000, 106:205-209.

18. Feudtner C, Hays RM, Haynes G, Geyer JR, Neff JM, Koepsell TD: Deaths attributed to pediatric complex chronic conditions: national trends and implications for supportive care services. Pediatrics 200I, 107:E99.

19. Stata Statistical Software: Release 7.0. College Station, TX: Stata Corporation 2000.

20. Feudtner C, Christakis DA, Zimmerman FJ, Muldoon JH, Neff JM, Koepsell TD: Characteristics of deaths occurring in children's hospitals: implications for supportive care services. Pediatrics 2002, 109:887-893.

\section{Pre-publication history}

The pre-publication history for this paper can be accessed here:

http://www.biomedcentral.com/1741-7015/1/3/prepub 